\title{
PHYLOGENY AND EVOLUTION
}

\author{
WALTER A. MARÍN \\ Escuela de Biología, Universidad de Costa Rica \\ wmarin@biologia.ucr.ac.cr
}

A particular human drive is to arrange things into categories, look for patterns and allocate labels. Since ancient times, humans have applied this to life itself. Today we use the Linnaeus' system of classification, first published in 1735 , which classifies living organisms by grouping them with the most similar visible characteristics into groups. This kind of scheme is called taxonomy. The system is based on groups nested within groups of increasingly general scope. So several species can belong to the same genus, several genera to the same family, and so on through order, class, phylum, and kingdom. Phenetic classification is an extension of the Linnean approach. According to the Phenetic method, species are grouped together with other species that they most closely resemble phenotipically.

The publication of Darwin's On the Origin of Species in 1859 led to a new approach to classification that attempted to unravel the evolutionary history of species. The closest species in this scheme are not necessarily those that show more resemblance, but those which share the most recent common ancestry.

The development of cladistic techniques, combined with the usage of computers to analyze large quantities of data, have produced new insights into the history of life. Cladistic classification determines the evolutionary relationships between organisms by analyzing certain kinds of characteristics, or traits. In the course of evolution, a novel, heritable trait will emerge in some organism. That trait will be passed on to its descendants. Two organisms that share such a new, or derived, trait or group of traits are therefore more closely related to each other than to organisms that lack those traits. By treating recently evolved characteristics differently from ancestral characteristics, this technique emphasizes evolutionary relationships over structural or morphological similarities.

Cladistics is now accepted as the best method avail- able for phylogenetic analysis. It provides an explicit and testable hypothesis of organismal relationships. The basic idea behind cladistics is that members of a group share a common evolutionary history, and are closely related, more so to members of the same group than to other organisms. These groups are recognized by sharing unique features which were not present in distant ancestors.

Cladistic analysis has become synonymous with phylogenetic systematics. A clade, a monophyletic taxon or evolutionary branch, is a group of organisms which includes the most recent common ancestor of all of its members and all of the descendants of that most recent common ancestor. The name clade derives from the Greek word "klados", meaning branch or twig. A taxon is monophyletic if a single ancestor gave rise to all species in that taxon and to no species in any other taxon. On the other hand a taxon is polyphyletic if its members are derived from two or more ancestral forms not common to all members, in this case the group does not include the most recent common ancestor of those organisms; the ancestor does not possess the character shared by members of the group.

Cladistic analysis uses a concept called outgroup comparison to recognize primitive characters for all members of a group of interest and to establish a starting point for a phylogenetic tree. An outgroup is a species or group of species. All members of the study group are compared as a group to the outgroup. Characters common to both the outgroup and the study group are likely to have been present in a common ancestor and are therefore shared primitive characters.

An important assumption of Cladistics is that characteristics of organisms change over time. It is only when traits change that it is possible to recognize different lineages or groups as well as the direction in 
which characters change, and the relative frequency with which they change. It is also possible to compare the descendants of a single ancestor to look at patterns of origin and extinction in these groups, or to look at relative size and diversity of the groups.

Cladistics is today the most commonly used method to classify organisms because it recognizes and employs evolutionary theory.

In recent years, DNA sequencing methods have become an useful tool for determining evolutionary relationships The DNA present in the cells of all living organisms provide a distinctive genetic profile of the species. By comparing the similarity of DNA between two species, scientists can determine how closely they are related. These molecular similarities reveal the relationships among organisms. The study of ancestral relations among species, often illustrated with a "tree of life" branching diagram, is also known as a phylogenetic tree.

In any species, usually there will be several variants, or alleles, of each gene. The alleles of a specific gene are related to each other - new alleles arise from older ones mostly by mutation. Molecular biologists can work out these relationships and draw up family trees that show which alleles are most closely related. When new species evolve from this ancestral population, some of the alleles may be lost through genetic drift or natural selection

The studies of molecular phylogeny have provided new ways for measuring how closely or distantly related different species are on the evolutionary tree. These molecular tools have greatly aided evolutionary biologists in tracing ancestor-descendant relationships, which show how later organisms developed from earlier ones, among various organisms on the tree of life.

The study of Fossils is mostly based on the analysis of morphological features that can be misled by incomplete evidence. The molecular techniques on the other hand detect differences in genes between organisms, which provide another kind of evidence to help determine how closely related they are. This allows the researchers to represent evolutionary relationships on a phylogenetic tree by the extent to which the organisms' gene sequences differ from each other. In fact, the genome, or entire set of genes, of an organism contains a record of the organism's evolutionary history.

The combination of information from analysis of fossils with DNA studies, allows researchers to draw evolutionary trees showing where and approximately when the species branched off from common ancestors. These diagrams, known as phylogenetic trees, or cladograms, are hypotheses that represent the best estimate of the true evolutionary relationships, based on existing evidence. They are constantly revised as new data become available

While molecular analysis has become a standard method of tracing evolutionary relationships, it also has limitations, and sometimes it can yield wrong answers. When genetic measurements and evidence from fossils conflict, scientists recognize that no one technique is always correct.

The best results obtained in evolutionary studies often come from combining analyses of the comparative morphology of different species with molecular techniques. The coupling of these approaches permit evolutionary biologists to build a more accurate picture of life's history.

\section{BIBLIOGRAPHY}

Dodson, E.O. and P. Dodson. 1985. Evolution: Process and Product. Prindle, Weber \& Schmidt Publishers. Boston.

Freeman, S and J. C. Herron. 2001. Evolutionary Analysis. Prentice-Hall Inc. New Jersey.

Kenrick, P. and P.R. Crane. 1997. The origin and early diversification of Land Plants: A cladistic study. The Smithsonian Institution Press. Washington.

Mayr, E. 2001. What evolution is. Basic Books (Perseus Books Group). New York.

Niklas, K.J. 1997. The evolutionary biology of plants. The University of Chicago Press. Chicago

Wilson, A.C. 1985. The molecular basis of Evolution. Scientific American 253:164-173.

Walter A. Marín, Ph.D (Physiological Plant Pathology, University of London) is Professor of Plant Anatomy and Plant Physiology at the Escuela de Biología, Universidad de Costa Rica. Currently conducting research on morphological study of organogenesis of maize in vitro and effect of water stress and fungal infection on growth and survival of seedlings of woody species. 